\title{
Innovations
}

\section{Clinical international experiences during medical school: Medical students' perspective}

\section{Authors}

\section{Ahmad N. Alkhaledi;; Ola A. El Kebbi²; Ahmed M. Saleh ${ }^{3 *}$}

\section{Abstract}

Migration and cultural interconnection has been one of the major contributors to globalization of disease. This is often a challenge physicians face as they encounter illnesses that they have not seen before while working in dissimilar environments to their training. Given this, international experiences are trending among medical professionals to be cognizant and proficient in dealing with such situations. In this paper, we will briefly reflect on our international exchange experiences as students and how such experiences can contribute to developing stronger global health knowledge and enrich our medical education experience as future physicians. We will also discuss some of the challenges medical students face while pursuing such opportunities, and how such challenges could be overcome.

\section{Keywords}

medical education; medical students; international experience

\section{Introduction}

Migration and cultural interconnection have been major contributors to the globalization of disease. This is a challenge physicians face as they encounter illnesses that they have not seen before while working in dissimilar environments to those where they trained.' Given this, gaining international experience is becoming popular among medical professionals who want to be cognizant of and proficient in dealing with such situations. In this paper, we will briefly reflect on our international exchange experiences as students and how such experiences can contribute to developing stronger global health knowledge and enrich our medical education experience as future physicians. We will also discuss some of the challenges medical students face while pursuing such opportunities, and how such challenges could be overcome.

International experiences are a great way to gain exposure to medical specialties that are often not frequently encountered in one's home environment. An example of this is highlighted in rotations such as tropical medicine, which adds to a student's experience in managing rare infectious diseases that are generally not seen in high-income countries,
'Faculty of Medicine, Damascus University. Damascus, Syrian Arab Republic.

${ }^{2}$ American University of Beirut. Beirut, Lebanese Republic.

${ }^{3}$ Weill Cornell Medicine - Qatar, PO Box 24144, Doha, Qatar.

*Email: ahs2006@qatar-med.cornell.edu

Ahmad N. Alkhaledi (iD) orcid.org/0000-0002-6128-0515

Ola A. El Kebbi (iD orcid.org/0000-0002-5538-1694

Ahmed M. Saleh (iD) orcid.org/0000-0001-7367-6616
Cite this article as:

Alkhaledi A., Kebbi OE, Saleh AS. Clinical international experiences during medical school: Medical students' perspective. Innovations in Global Health Professions Education. 2018:1. http://dx.doi.org/10.20421/ighpe2018.01

This is an open access article distributed under the terms of the Creative Commons Attribution license CC BY 4.0, which permits unrestricted use, distribution and reproduction in any medium, provided the original work is properly cited. 
either due to the differing environmental conditions or the availability and accessibility of vaccinations and prophylactic medications. ${ }^{1-3}$ Additionally, during international experiences physicians and medical students may be exposed to cases involving increased severity of a disease, which is typically the case in medically underserved areas due to the inaccessibility of healthcare. Furthermore, common traditions and beliefs may drive patients to seek initial healthcare from traditional healers, which also leads to late hospital presentation with more complications.

Students are given the chance to adapt to different ways of practicing medicine, as they have to adapt to a new environment and use the available resources. Many opportunities occur in low resource settings where laboratory and radiological workup is not always accessible. This encourages students to depend heavily on their history-taking skills and physical examinations to come up with a diagnosis and treatment plan, and provides a great opportunity for students to strengthen their clinical reasoning abilities and valuable bedside skills. ${ }^{3-6}$

International experiences also expose students to different medical systems and cultures that they might not be familiar with. This encourages students to think about how medicine is practiced elsewhere and identify some positive differences that they may be able to implement back home. Examples of this include different ways of strengthening the doctor-patient relationship, communication skills, and getting a better understanding of social determinants of health and how such factors affect patients' well-being in various settings.?

Language and communication are essential aspects of international clinical experiences. Students often pursue experiences in locations where they don't speak the local language, which makes it more difficult to communicate with patients and healthcare workers. This adds another level of complexity to the experience as students need to find a way to connect, which is often sought through either medical interpreters or by learning the essential terms to conduct a basic history and physical exam.

Another benefit of international experience is that students can often gain exposure to additional specialties that they were unaware off. This may help students to steer their future career and postgraduation plans, either in choosing a specialty of interest or a specific geographic location where they'd want to practice in the future. ${ }^{7}$ Additionally, these international experiences help initiate new connections and build bridges between healthcare providers from across the world, which supports clinical and research collaborations. ${ }^{7}$

\section{Our experiences: Ahmad Alkhaledi, from Syria to Lebanon}

\section{A different healthcare system}

Culture shock is often something people face while traveling to new places that are culturally dissimilar to one's origin. Surprisingly, coming to Beirut from Damascus - two places that are just a couple of hours away from each other - had a similar effect on me as I was navigating a new healthcare system. I was faced with people, culture, and a language that are similar to my own while walking around Al-Hamra street in downtown Beirut. However, the underpinnings of the healthcare system at the American University of Beirut Medical Center (AUBMC) were somewhat different from those at Damascus University.

The internal medicine department's ward, 5-South, was where I started. The way physicians and allied healthcare workers communicated with patients surprised me. The big role and active involvement of medical students and interns in managing patients was one thing that I wasn't quite familiar with. As a 5th year medical student at Damascus University, a six-year program, I was never given the chance to be the primary person taking care of a patient, starting from taking history, performing physical examinations, coming up with a differential diagnosis list, all the way to presenting the patient to my team, and coming up with a management plan.

AUBMC uses the American style in teaching medical students, in which both 3rd and 4th year medical students take care of patients and are actively involved in their management. Students write notes, present patients, and follow up on orders under the supervision of residents and attending physicians. This concept was new to me, the entire experience of rounding on the wards twice daily, reporting every 
detail to the superiors, and writing notes was initially difficult but extremely valuable. And to make it even harder, I struggled as I was trying to adapt to using English as the primary language of instruction and communication (Damascus University uses Arabic as their teaching language, including medical terminology). This whole experience was tough, but has taught me endless lessons on the role of medical students in a markedly different healthcare system.

My time at AUBMC has added a lot to my experience as a student. It taught me that being a doctor needs more than just studying and reading up on different diseases and treatments. It requires supervised practice to ensure the mastery of the skills you acquire during medical school. I worked on integrating myself into the system by practicing with other medical students to learn the terminology and how everything got done on the wards. I wrote many templates that I could utilize while writing admission notes and practiced presenting patients to my fellow students. Later during the elective, I started presenting patients to the team as I got used to the new system. During my elective I realized how international rotations can add to your experience as a student and a clinician. I have carried these skills with me back home and have helped my home institution incorporating such practices in the students' daily routine, which is rewarding and humbling.

\section{Our experiences: Ola El Kebbi, from Lebanon to Turkey}

\section{Empathy across borders}

Following my first year of medical school, I was given the opportunity to participate as a research assistant at Bezmialim Hospital in Istanbul. Weeks before my departure date, I started packing while thinking of the potential medical knowledge that I will gain out of this experience. I was excited about all the possible adventures I would have and that I would finally make use of the Turkish lessons I had taken on Duolingo. ${ }^{8}$

Upon my arrival, I was assigned to shadow Dr. Hatibolgu, the chief neurosurgeon. My time was divided between polyclinic days, gamma knife days and surgery days. As a first year medical student with only basic knowledge of neuroanatomy, I cannot say that I mastered it all in a month. However, scrubbing into brain surgeries, watching the consummate process of putting gamma knife frames and, most importantly, the time spent at the polyclinic taught me something I could only learn abroad in a place where I could barely communicate: empathy.

Bezmialim Hospital is a foundation hospital built during the Ottoman era to meet the needs of people who may not be able to pay for their medical treatment. The hospital requires only reduced fees, and in some cases, none at all. Polyclinic days were definitely a marathon of seeing patients endlessly pooling into the clinic from the huge overcrowded waiting room. Those patients never came in alone, they were usually cancer patients who walked in with multiple family members, and everyone was highly stressed. Dr. Hatibolgu always made sure that his assistant got extra chairs in the room, so that everyone could have a seat. He leaned in and maintained eye contact with his patients, and I could tell that he asked not only about their medical condition but also about their overall wellbeing as those warm Turkish phrases were all that I knew. Most importantly Dr. Hatibolgu never rejected a patient's request to take a look at some random labs or radiology images that he knew are not related to their case, even if it meant that he had to spend his lunch break reassuring them. He would keep a soft smile throughout the visit, and by the end of the consultation you could tell, without understanding a single word, that his patients and their families are calmer, happier, and satisfied. They would all leave while repeating phrases of thankfulness to God and appreciation to Dr. Hatibolgu: "Teşekkür ederim, Tanrıya şükür". He would then explain to me the rather morose diagnosis.

Sitting in a clinic for hours where I can only understand the warm Turkish greetings and observe the body language and facial expressions of Dr. Hatibolgu and his patients highlighted the importance of empathy when communicating with patients. During my school's clinical communication session, they lectured us on how to show empathy to our patients. We are taught to make eye contact, lower our voices, have the same seating level as the patient when addressing them, stand to their left side when they are lying in bed, and so on. Unfortunately, those 
tips usually do not leave the classroom as we later focus on getting to the precise technical and clinical diagnosis and ignore the fact that we are being emotionally detached. After my time with Dr. Hatibolg, I realized that those tips should be taken seriously as they do make a significant difference.

My experience abroad taught me that empathy is what draws the line between a doctor who treats the disease and another who heals. Having a solid medical background may treat the patient's illness, but being aware of his emotional needs and meeting them will heal him/her and that is what medicine is all about. An excellent physician should have not only knowledge, experience and discipline, but also empathy. My time in Turkey taught me that foreign experiences in medicine come with a myriad of personal and academic benefits. While most medical students return to their home countries with a new perspective after being exposed to different medical systems, cultures and languages, my experience in Istanbul has given me an appreciation of the importance of empathy in medicine.

\section{Our experiences: Ahmed Saleh, from Qatar to Italy}

\section{When the doctor doesn't speak the language}

My first day at the Maugeri Fondazione hospital in Pavia, Italy, felt just like any other "first day at a new hospital" in a medical student's life: feelings of uncertainty and being lost. Doctors and nurses spoke enough English for me to follow what was going on, but I realized the day wasn't that typical once we started rounding and talking to patients. Before my time in Pavia, I had never had issues communicating with patients as l'd either speak their language or I had access to instant interpretation services over the phone through the hospitals where I rotated. Obtaining a history, performing physical exam maneuvers, and explaining the diagnosis and treatment plan to patients was easily manageable. However, my rotation in Italy made me realize how challenging it could be to provide high quality, safe and culturally competent care to patients when you do not speak their language.

My experience in Pavia has helped me gain a different perspective on what works well and what does not when practicing medicine if you don't speak the same language as your patients. I remember spending a lot of time discussing the importance of communication in healthcare during my medical school courses; whether it's communication among physicians and healthcare workers or between healthcare workers and patients and their family members. It is often said that physical exams and diagnostic workups are of no use in the absence of a focused, accurate and complete history. Weill Cornell Medicine - Qatar provided us with ample opportunities to work with diverse populations and be culturally competent in theory, however, applying such skills isn't always as easy as you might expect.

My international clinical experience has provided me with useful tips that could be utilized in such situations. For instance, it is always useful to learn some key phrases and medical terms in the language of the country where you are rotating. It might not be feasible to learn a whole new language or be able to carry a conversation, but learning some basic terms to help you communicate with your patients to obtain a basic history and perform a quick physical exam is worthwhile: phrases such as "take a deep breath", "stop breathing", "lie down", and "look left or right" are all advantageous, and make performing a physical exam less challenging. Additionally, it is important to speak slowly and calmly, even when someone is interpreting. I learned the importance of non-verbal communication via body language and eye contact to deliver and receive information, and how these play a major role in building a strong doctor-patient relationship even when both do not speak the same language.

These tips will definitely not the solution to eliminate all confusion or uncertainty that might arise, but keeping them in mind helps. My time in Pavia was definitely an eye opener and I started considering communication issues on a deeper level and how they affect the doctor-patient relationship and ultimately patient outcomes. Medicine is all about making sure that patients are getting the best of culturally competent patient-centered care with no barriers, and language barriers should always be addressed efficiently.

\section{Conclusion}

Previous research has highlighted the benefits of international experiences for medical students. 
Medical students who participate in international clinical rotations during their studies cope more easily when practicing medicine later on in their career working with the underserved. 2,3 Additionally, those who participate in international clinical rotations are more likely to choose a primary care track, and practice medicine in underserved locations with multicultural populations. ${ }^{2,3}$ Resident physicians who participated in international rotations as students have also reported developing a deeper appreciation of public health issues and developing better cultural sensitivity. ${ }^{4,7}$ Students who are given the chance to participate in research projects during their international experiences gain valuable research methodology skills. ' Students are often exposed to new ways of conducting research and tend to generate research questions that are specific to their particular setting.

Students are often faced with multiple obstacles and challenges before and during their international experiences. Finances are always the major challenge students face, as such experiences often cost a significant amount of money to cover transportation, visa costs, housing, living expenses, course fees, malpractice insurance, and health insurance. Some international organizations and medical schools provide students with some meritbased scholarships and financial support to cover their international experiences. More support and funding should be directed towards such means of support, and more advertising is needed to make sure students are aware of such resources.

Additionally, some medical students face difficulties as they navigate the new health care system and adapt to a new environment. This may be due to a lack of support from both the sending and hosting institutions. Some institutions are assigning faculty and staff mentors to help students and guide them through their experience. Additionally, some programs have implemented student ambassador programs where students at the host institution serve as points of references for any incoming student.

Our experiences as exchange students highlight the benefits of seeking international experiences during medical school. The lessons we have learned have impacted the way we see healthcare and our understanding of how medicine is practiced elsewhere around the world. Additionally, international experience has broadened our clinical knowledge and strengthened our skills in communication and critical thinking. We believe medical schools should encourage and support international experiences with the provision of elective time and financial support. Lastly, we strongly urge medical students to consider enrolling in such learning opportunities early on in their career given the great benefit they could gain from such experiences.

Acknowledgements: We would like to acknowledge and thank Dr. Sahar Koubar at the Department of Internal Medicine at the American University of Beirut (AUB), Dr. Arafat Tfayle and Dr. Deborah Mukerii at the AUB Department of Oncology and the Naef K. Basile Cancer Institute of AUB. We'd also like to acknowledge the Department of Neurosurgery at Bezmialim Vakif University and Dr. Mustapha Aziz Hatibolgu and Bezmialim Hospital's staff and faculty. We'd also like to acknowledge Weill Cornell Medicine-Qatar's Institute for Population Health, University of Pavia's Medical School, Salvatore Maugeri Foundation's Nephrology Department and Global Educational Exchange in Medicine and the Health Professions- GEMx for their great support and guidance throughout our experiences.

\section{Conflicts of interest: None.}

Funding sources: Ahmed M. Saleh has received partial funding through the Global Educational Exchange in Medicine and the Health ProfessionsGEMx to support his international experience.

\section{References}

1. Cherniak WA, Drain PK, Brewer TF. Educational objectives for international medical electives: a literature review. Acad Med [Internet]. 2013 [cited 2018 Apr 12]; 88(11):1778-81. Available from: https:// journals.Iww.com/academicmedicine/ Fulltext/2013/11000/Educational Objectives for International Medical.49.aspx doi: 10.1097/ ACM.0b013e3182a6a7ce

2. K, Primack A, Hunt DD, Fawzi WW, Holmes KK, Gardner P. Global health in medical education: a call for more training and opportunities. 
Acad Med. 2007 Mar;82(3):226-30. doi: 10.1097/ACM.0b013e3180305cf9.

3. Gupta AR, Wells CK, Horwitz RI, Bia FJ, Barry $M$. The International Health Program: the fifteen-year experience with Yale University's Internal Medicine Residency Program. Am J Trop Med Hyg. 1999 Dec;61(6):1019-23. doi: 10.4269/ajtmh.1999.61.1019.

4. Miller WC, Corey GR, Lallinger GJ, Durack DT. International health and internal medicine residency training: the Duke University experience. Am J Med. 1995 Sep;99(3):291-7. doi: 10.1016/S0002-9343(99)80162-4.

5. Barry M, Bia FJ. Departments of medicine and international health. Am J Med. 1986 Jun;80(6):1019-21. doi: 10.1016/00029343(86)90658-3.

6. Smilkstein G, Culjat D. An international health fellowship in primary care in the developing world. Acad Med. 1990 Dec;65(12):781.

7. Haq C, Rothenberg D, Gjerde C, Bobula J, Wilson C, Bickley L, et al. New world views: preparing physicians in training for global health work. Fam Med. 2000 Sep;32(8):56672.

8. Duolingo [Internet]. Pittsburg, PA: Duolingo; 2011 - [cited 2018 Apr 12]. Available from: https://www.duolingo.com/ 